\title{
Diseño De Instrumento Para Evaluar Clima Organizacional De Universidades Públicas En México
}

\author{
Hortensia Hernández Vela \\ Norma Patricia Garrido \\ Ma. Concepción Rico Pérez
}

Facultad de Economía, Contaduría y Administración de la Universidad Juárez del Estado de Durango, México

doi: 10.19044/esj.2016.v12n28p312 URL:http://dx.doi.org/10.19044/esj.2016.v12n28p312

\begin{abstract}
The measuring of organizational climate aims to obtain feedback that can inform an organization on ways in which to make improvements; not only in the work environment, but also in the quality of it; hence, it is important to possess instruments that provide reliable information of what is intended to be measured. Describing the building process of an organizational climate instrument is the objective of the present article, in which, after having established the relevance of the topic in the introduction; the stages developed for the elaboration and validation of the measuring instrument of the organizational climate perceived by professors of Higher Education Institution are presented. The result of this process is an instrument with four factors and nineteen dimensions.
\end{abstract}

Keywords: Organizational climate, measuring instrument, university professor

\section{Resumen}

La medición del clima organizacional, tiene por objeto retroalimentar a la organización para mejorar, no solamente el ambiente de trabajo sino la calidad del mismo, de ahí que sea importante contar con instrumentos que nos arrojen una información confiable de lo que se pretende medir, la descripción de la construcción de un instrumento para clima organizacional es el objetivo de este artículo, en donde una vez establecida la importancia del tema en la introducción, se presentan las etapas desarrolladas para la elaboración y validación de un instrumento de medición del clima organizacional que perciben los docentes de una Institución de Educación 
Superior, siendo el resultado del proceso un instrumento con cuatro factores $\mathrm{y}$ diecinueve dimensiones.

Palabras clave: Clima organizacional, instrumento de medición, docentes universitarios

\section{Introducción}

El clima es un tema que ha sido ampliamente abordado en las organizaciones y no es ajeno a las instituciones de educación superior, en las que se aborda desde perspectivas diferentes y siempre bajo la premisa de que la medición es puntual, es decir bajo el contexto que prevalece, en el momento de la evaluación.

Normalmente se considera que hay un solo clima en toda la organización, pero las Instituciones de Educación Superior (IES) en particular están conformadas por partes flojamente acopladas como son por un lado las diferentes escuelas que las conforman, las áreas administrativas y de éstas los estratos de alumnos, docentes y personal administrativo y aunque la IES es un todo, cada parte conserva su propia identidad (Weick, 1975), bajo estas premisas, este trabajo describe el diseño de un instrumento para medir el clima organizacional que perciben los docentes de una universidad pública.

La importancia de conocer el clima radica según Mujica de González y Pérez de Maldonado (2007, p.292), en que “el clima o ambiente de trabajo constituye uno de los factores determinantes, no sólo de los procesos organizativos y de gestión, sino también de transformación”, para éstas autoras (2007, p.293) “la gestión del clima organizacional en los diferentes niveles de la universidad, hoy por hoy, constituye un compromiso y una responsabilidad para los directivos universitarios que deben actuar con determinación para establecer la calidad como valor fundamental que ha de incorporarse a las normas administrativas de la organización”.

La medición del clima, tiene por objeto realimentar a la institución para mejorar no solamente el ambiente de trabajo sino la calidad del mismo, de ahí que sea importante contar con instrumentos que nos arrojen una información confiable de lo que se pretende medir, que en el caso que nos ocupa es el Clima Organizacional en Instituciones de Educación Superior Públicas en México, acorde a su realidad, que brinde la oportunidad a los docentes de externar su opinión en relación a las diversas dimensiones que afectan al Clima, para considerar a estas dimensiones como indicadores a partir de los cuales se puedan establecer estrategias puntuales de intervención, en este sentido (García, Hernández, Navarrete, Corichi y Sánchez, 2014) señalan que si bien son numerosas las dimensiones que se presentan en diferentes modelos es importante considerar que el instrumento 
de evaluación más recomendable o conveniente utilizar, es aquel que incluya la información que permita medir realmente lo que le interese identificar al investigador.

\section{Método}

Para elaborar un instrumento que cumpla con este objetivo se realizaron las siguientes etapas:

- Primera etapa: construcción del Modelo de Clima Organizacional

- Segunda etapa: revisión de investigaciones sobre clima organizacional en las IES

- Tercera etapa: revisión de instrumentos sobre clima organizacional

- Cuarta etapa: análisis de la pertinencia y selección de las dimensiones para el Modelo de la IES

- Quinta etapa: incorporación de las dimensiones al modelo de clima organizacional establecido en la primera etapa.

- Sexta etapa: revisión y adaptación de ítems e integración del instrumento

- Séptima etapa: prueba piloto

- Octava etapa: aplicación del instrumento, calificación de las respuestas e interpretación

- Novena etapa: determinación de la consistencia interna

- Décima etapa: determinación de la validez del modelo

\section{Marco Teórico}

Las etapas primera segunda y tercera, corresponden al sustento teórico del instrumento, en la cuarta, quinta y sexta etapas se realiza la validez de contenido del instrumento, en la séptima y octava etapas se realiza la prueba y aplicación del instrumento, en la novena etapa se calcula la consistencia interna (confiabilidad) y en la décima etapa se prueba la validez del modelo (validez del constructo). Las etapas el marco teórico en el que se basó el instrumento consideran primero (Etapa 1) a diversos autores que han analizado esta temática, para continuar con los que específicamente la han abordado para las IES (Etapa 2) y la revisión de os instrumentos generados de éstas propuestas (Etapa 3).

Primera etapa: Construcción del Modelo de Clima Organizacional y conceptualización de Clima en las Organizaciones. El modelo que se construyó para las IES; tiene como base el trabajo de Marlenis Ucros Brito (2011), en la que compara y analiza los diferentes enfoques y sus dimensiones psicológicas, grupales y organizacionales sobre clima organizacional reseñados por Likert $(1961,1967)$, Litwin y Stringer (1968), Schneider (1968, 1983), Payne (1971), Jones et al. (1979), Silva (1992), Álvarez (1992), Brunet (1987), Goncalvez (2000), Toro (2005), 
Rodríguez (2005) y Ménde (2006). La autora clasifica las dimensiones propuestas por los autores señalados de

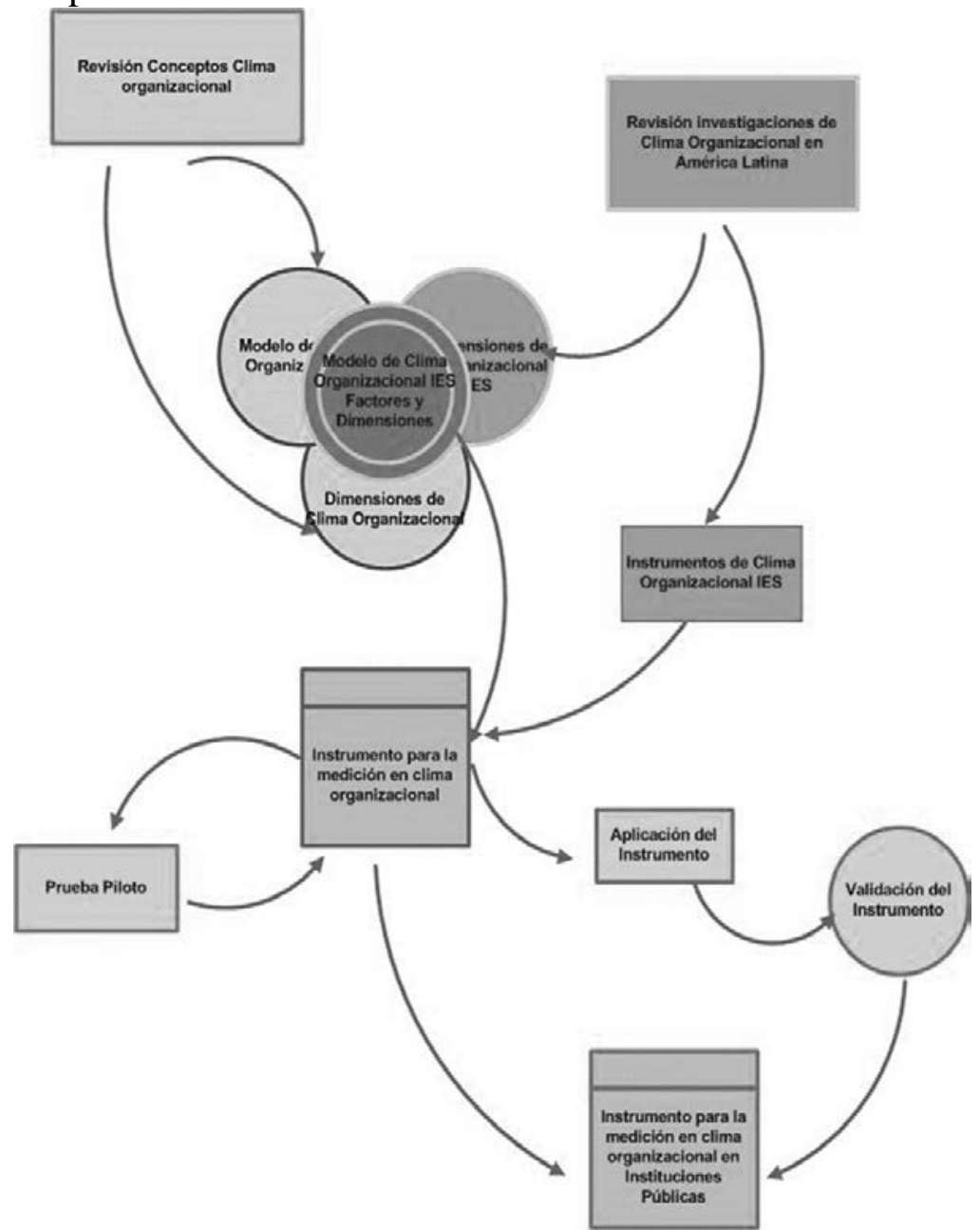

Figura 1. Etapas para la elaboración del Instrumento para medir clima organizacional en IES.

Elaboración propia acuerdo a los tres tipos de factores propuestos por Brunet (1987): Factores psicológicos individuales, factores grupales y factores organizacionales.

- Los Factores Psicológicos Individuales incluyen los procesos psicológicos, entre los que destacan: a) la necesidad del individuo de establecer interacción social, b) necesidad de establecer sentimientos de afinidad hacia la organización y c) construcción de un sentimiento positivo o negativo al sentir pertenencia a la organización (Schneider 1968, citado por Ucros, 2011). También se incluye el sentir y la manera de reaccionar de las personas frente a las características de cada organización y ante diferentes situaciones, según sus construcciones personales de significados. Son 
factores psicológicos individuales la autonomía, el grado de satisfacción, la motivación, el sentido de pertenencia, compromiso y lealtad con los objetivos, responsabilidad en el desempeño y la disposición al cambio.

- $\quad$ Los Factores Grupales se establecen a partir de la propuesta de Toro (2003) quien define al clima como "la percepción colectiva y compartida de las realidades internas del grupo donde los aspectos sociales de la tarea se convierten en una fuente de satisfacciones permanentes, se genera crecimiento personal, se aprende a ser tolerante, a cooperar, a respetar las diferencias...”, son factores grupales el espíritu de colaboración, la confianza en el jefe; las relaciones interpersonales, el desarrollo del trabajo en equipo, liderazgo y valores colectivos.

- $\quad$ Los Factores Organizacionales son los factores objetivos o atributos de la organización que afectan el clima organizacional, entre los cuales algunos autores destacan: comunicación y difusión de políticas, modelo de toma de decisiones, estructura organizacional, nivel jerárquico y su influencia en el cargo, riesgos laborales e institucionales, infraestructura física, equipamiento tecnológico y dotación de insumo docente.

El análisis de los factores organizacionales, derivó en la conceptualización de dos tipos de factores:

- Los Factores Organizacionales Objetivos que eran aquellos que se espera sean percibidos de manera semejante bajo una misma cultura y contexto y

- $\quad$ Los Factores Organizacionales Subjetivos que están relacionados a percepciones subjetivas, como es caso de sueldos y salarios. Quedando el Modelo del clima organizacional en las IES, con formado por factores: personales, grupales, organizacionales objetivos y organizacionales subjetivos.

En esta etapa se revisaron también las dimensiones incluidas para la medición del clima señaladas por los autores clásicos y se definieron cada una ellas en base a equivalencias entre los diversos autores.

Segunda Etapa: revisión de investigaciones sobre clima organizacional en las IES. Se realizó una selección de investigaciones sobre clima organizacional realizadas en América Latina y que contaban con un instrumento válido, confiable y aplicado en instituciones de Educación, aclarando que todas ellas se enfocaban a la evaluación del clima a través de los docentes, como primer paso, se realizó una revisión con el fin de clasificar los conceptos sobre clima organizacional incluidos en los estudios, tanto las posturas originales de los autores como las de los autores citados por ellos.

En seguida se procedió a identificar las dimensiones que habían sido consideradas por cada uno de los 23 autores que se analizaron, quedando como dimensiones: autonomía individual, motivación, identidad y 
pertenencia, trabajo significante, logro, calor y apoyo, valores colectivos, solución de conflicto, trabajo en equipo, estructura, comunicación, claridad y objetivos organizacionales, infraestructura, innovación, salario y recompensas, liderazgo, capacitación y desarrollo, supervisión, estabilidad, participación, desempeño, riesgo, tolerancia y apertura.

Estas dimensiones eran mencionadas y definidas por uno o más de los autores encontrando equivalencias, en las definiciones señaladas, y a partir de ellas se elaboró una definición para cada dimensión, que integró los conceptos de los autores consultados.

Tercera etapa: revisión de instrumentos sobre clima organizacional. Solamente siete investigaciones, de las que se revisaron contaban con un instrumento para la medición del clima organizacional en las IES a través de la opinión de los académicos siendo éstas:

El trabajo de Emig y Lazo (2002) denominado Clima organizacional: Estamento Académico de la Universidad Austral de Chile, tuvo como objetivo general analizar el Clima Organizacional existente en el estamento académico de la Universidad Austral de Chile, y de manera específica, determinar el grado en que las dimensiones en estudio afectan el Clima Organizacional. El instrumento utilizado corresponde al aplicado por Redlich y Trautmann (1996) en la tesis "Un estudio aplicado de clima organizacional en el Estamento no académico de la Universidad Austral de Chile, sede Valdivia” adaptación de los cuestionarios de Clima Organizacional de Litwin y Stringer (1968), Newman (1977) y Astudillo (1985). Este instrumento considera catorce dimensiones que explicarían el Clima Organizacional de la institución: Estructura, responsabilidad, recompensa, riesgo, calidez, apoyo, administración del conflicto, identidad, estilo de supervisión, motivación laboral, estabilidad laboral, oportunidades de desarrollo, comunicación y equipos y distribución de personas y material.

Chiang, Nuñez y Huerta (2007) realizaron el estudio Relación del clima organizacional y la satisfacción laboral con los resultados, en grupos de docentes de instituciones de educación superior con el objetivo de establecer la posible relación entre las variables del clima organizacional y de la satisfacción laboral con los resultados obtenidos por profesores y/o investigadores que trabajan en un departamento de una universidad. Las dimensiones para medir el clima organizacional fueron: libertad de cátedra, interés por el aprendizaje del estudiante, interés por la investigación y el estudio, empowerment, afiliación, consenso de la misión y presión laboral. El instrumento fue desarrollado por Chiang, (2004) a partir del ULEQ University-Level Environment Questionnaire de Dorman (1998).

El trabajo realizado por Salaiza (2008) denominado Validez Factorial de la Encuesta de Clima Laboral de Ricardo Valenzuela, analiza la validez y confiabilidad de un instrumento que mide el clima laboral El instrumento 
mide el clima laboral a través de 10 dimensiones: trabajo personal, supervisión, trabajo en equipo y relaciones con compañeros de trabajo, administración, comunicación, ambiente físico y cultural, capacitación y desarrollo, promoción y carrera, sueldos y prestaciones, y orgullo de pertenencia.

El estudio realizado por Acosta y López de Maldonado (2010) denominado, Diagnóstico Clima Organizacional, Colegio Gran Bretaña, Concepción. En este estudio, se pretendió despertar la reflexión de la dirección de la organización educativa, sobre las condiciones laborales en las que se desenvuelve el personal administrativo, y docente, considerando que la cultura y el clima organizacional son factores determinantes en la eficacia administrativa tomando en cuenta además que el comportamiento del grupo está condicionado por la percepción que tenga éste de la organización. En los factores de la relación educativa se consideraron las siguientes dimensiones: respeto, confianza, motivación, oportunidades para aportar, crecimiento académico y personal, identificación institucional y atención personalizada. En los factores del proceso educativo se incluyeron las dimensiones de: solución adecuada de problemas, constante ajuste de metas, identificación y manejo de conflictos, comunicación efectiva, participación en la toma de decisiones, autonomía y planificación del futuro. En los factores materiales se incluyeron las dimensiones: recursos adecuados, sistemas de apoyo logístico y apropiada planta física.

En el estudio realizado por Diana Guadalupe Garza Puente (2010), denominado Análisis del clima organizacional. Caso de estudio de la Coordinación de Seguridad y Custodia del Centro de Ejecución de Sanciones de la Secretaría de Seguridad Pública en Tamaulipas, se buscó proponer recomendaciones que contribuyan a mejorar la percepción que los empleados de esta dirección tienen sobre el clima organizacional que prevalece en su área de trabajo. El instrumento diseñado midió 16 dimensiones: Autonomía, trabajo en equipo, apoyo, comunicación, presión, reconocimiento, equidad, innovación, percepción de la organización, motivación intrínseca, sueldos y salarios, promoción y carrera, capacitación y desarrollo, ambiente físico y cultural, visión y satisfacción general.

Chiang, Nuñez y Huerta. (2010) llevaron a cabo el estudio denominado Efecto del clima organizacional en la autoeficacia de los Docentes de instituciones de educación superior con el propósito de analizar las relaciones entre el clima organizacional y la autoeficacia, en grupos de trabajo formados por profesores y/o investigadores que trabajan en un departamento de una universidad. Para lograr los objetivos descritos se procedió a la aplicación del instrumento elaborado por Chiang, (2004) a partir del ULEQ University-Level Environment Questionnaire de Dorman (1998). Las dimensiones para medir el clima organizacional fueron: libertad 
de cátedra, interés por el aprendizaje del estudiante, interés por la investigación y el estudio, empowerment, afiliación, consenso de la misión y presión laboral.

Prisco y Salaiza (2012) en el estudio denominado Relación entre el clima laboral y la motivación del personal en una secundaria pública, buscaron analizar la relación entre el Clima Laboral y los factores de Motivación de una institución educativa. Se utilizaron dos instrumentos validados: La encuesta de Clima Laboral de Valenzuela (2004) y el cuestionario ¿Qué lo motiva a usted? basado en la teoría de motivación de Mc Clelland. Las dimensiones para medir el clima laboral fueron: trabajo personal, supervisión, trabajo en equipo y relaciones con compañeros de trabajo, administración, comunicación, ambiente físico y cultural, capacitación y desarrollo, promoción y carrera, sueldos y prestaciones, y orgullo de pertenencia.

El trabajo realizado por Diana Velázquez Ramírez (2012) denominado Clima Organizacional en la Universidad Del Valle, contiene el informe final del diagnóstico del clima organizacional realizado en la Universidad del Valle, cuyos resultados son manejados a través de conclusiones globales, involucrando a los estamentos pertenecientes a la Universidad y agrupándolos de la siguiente manera: Estamento Docente (docentes nombrados y contratistas), Estamento Administrativo (empleados públicos, trabajadores oficiales y contratistas estatales) y personal contratado por la Fundación. El instrumento fue elaborado a partir de los propuestos por Litwin y Stringer, Likert, Sudarsky (1977) y Álvarez Londoño (1993, cit. García, 2009). Las dimensiones consideradas en el instrumento fueron: Entendimiento de la política de calidad, estilo de la dirección, compensación, reconocimiento, condiciones de trabajo, recursos, relaciones interpersonales, retribución, compromiso, trabajo en equipo.

Los instrumentos empleados en cada una estas investigaciones fueron analizados por un grupo de investigadores del área, quienes trabajaron como grupo de enfoque para determinar las dimensiones en un modelo incluyente definiendo a cada una de ellas.

\section{Validez de contenido:}

En las etapas cuarta, quinta y sexta, se realizaron las actividades necesarias para medir la validez de contenido: se analizaron y seleccionaron las dimensiones y los ítems relevantes y representativos del clima organizacional, utilizando el método empleado por Lawshe (1975, citado por Cohen y Swerdlik, 2001) quien desarrolló la fórmula denominada razón de validez de contenido (CVR). Para cada reactivo, cada miembro del jurado responde si el contenido (dimensión, ítem) es esencial, útil pero no esencial o no necesario para medir la variable. En este caso, los jueces fueron cinco 
integrantes del Cuerpo Académico, “Procesos de Cambio y Desarrollo en las instituciones” perteneciente a la UJED.

De acuerdo con el método de Lawshe (1975), al validar una prueba, se calcula la validez de contenido para cada reactivo. De acuerdo al número de jueces (cinco en este caso) el valor mínimo de la CRV es de 0.99, por lo que se estableció que debía haber consenso en los jueces en la selección tanto de las dimensiones como de los ítems.

Cuarta etapa: análisis de la pertinencia y selección de las dimensiones para el Modelo para las IES. En esta etapa, el propósito fundamental fue determinar la representatividad de cada una de las dimensiones propuestas en las investigaciones seleccionadas en la etapa anterior para la medición del Clima Organizacional en las IES. Ante cada dimensión se estableció la pregunta ¿esta dimensión es esencial para la medición del clima en la IES? Y en base a los conceptos incluidos en la definición en las etapas 1 y 2 se seleccionaron sólo las dimensiones en las que hubo consenso entre los integrantes del cuerpo académico, quedando 19 dimensiones:

- Motivación Intrínseca. El grado de interés que siente el académico respecto a su trabajo debido a factores provenientes del mismo (variedad, identidad, significación, autonomía y auto retroalimentación).

- Identidad. El grado de conocimiento e identificación de los académicos con la organización y el orgullo de pertenencia, que los lleva a actuar con lealtad y compromiso hacia las metas y valores colectivos de la organización.

- Autonomía. Percepción de independencia o posibilidad de actuar y pensar, sin depender del deseo de otros en relación a los procedimientos de trabajo, los objetivos y las prioridades.

- $\quad$ Trabajo en equipo. Es la percepción del grado en que los miembros de la organización colaboran y cooperan entre sí, se apoyan mutuamente y mantienen relaciones de amistad y compañerismo. En síntesis, el trabajo en equipo es la percepción de los individuos sobre el compromiso, la sinergia, responsabilidad y las destrezas que los miembros de la organización manifiestan.

- Apoyo. Es la percepción que tienen los académico acerca del respaldo y tolerancia de su comportamiento dentro de la institución; esto incluye el aprendizaje de los errores por parte del académico, sin miedo a las represalias de sus superiores o compañeros de trabajo.

- Administración del conflicto. Percepción que tienen los académicos, acerca de la posibilidad de que la dirección de la institución propicie el que ellos manifiesten diferencias de opinión en ciertos aspectos 
del trabajo; y del grado en el cual, en el ambiente de trabajo, se dirimen las diferencias de opinión.

- $\quad$ Respeto. Percepción que tienen los académicos del trato digno que reciben como personas.

- $\quad$ Percepción de la organización. La imagen con la que el académico supone que es vista la institución.

- Visión. La percepción de los fines de la organización, que representan una fuerza motivacional para el trabajo. Es la fuente de inspiración de la organización, ayudando a trabajar por un motivo y en la misma dirección a todos los colaboradores de la institución.

- Comunicación. La percepción que tienen los académicos acerca de los canales de información que tiene la institución y de la forma en que fluye en los diferentes niveles jerárquicos, así como la libertad para comunicarse abiertamente con los superiores, para tratar temas sensibles o personales con la confianza suficiente de que esa información no será empleada indebidamente.

- Condiciones de trabajo. La percepción de los académico acerca del espacio en el cual se desenvuelve la vida diaria de la organización, que les permite realizar su trabajo con calidad y comodidad, (considerando condiciones tales como ruido, iluminación, color, música, humedad, temperatura, higiene y seguridad), en donde el equipo y la distribución de personas $\mathrm{y}$ del material permiten operaciones de trabajo eficientes $\mathrm{y}$ efectivas.

- Innovación. Percepción de los académicos sobre el ánimo y el apoyo por parte de la dirección para promover la creatividad de las personas y motivarlas a asumir riesgos en la búsqueda de nuevas formas de hacer su trabajo; pensando siempre en eficientar el tiempo y los recursos necesarios para la realización de sus actividades.

- Reconocimiento. Indica los sentimientos de los académicos de sentirse recompensados (felicitaciones, distinciones y reconocimientos personales) por un trabajo bien hecho en oposición a la crítica y el castigo.

- Sueldos y salarios. La percepción de justicia y equidad en el salario y compensaciones recibidas.

- Capacitación y desarrollo. Es la percepción que tienen los miembros de la organización acerca de las posibilidades reales y permanentes de continuar su formación personal y profesional, útil para el desempeño de sus actividades.

- Promoción y carrera. La percepción que los académicos tienen acerca del sistema de la institución, para que hagan una carrera en ella.

- $\quad$ Equidad. La percepción que los académicos tienen acerca de la existencia de políticas y reglamentos equitativos y claros dentro de la 
institución. No es sólo el equilibrio entre sus esfuerzos y las recompensas recibidas, sino también la relación que guarda este equilibrio con las recompensas que se les brindan a las otras personas por sus esfuerzos.

- Presión. Percepción que tienen los académicos en relación a la exigencia de trabajo que la organización coloca sobre ellos (carga de trabajo/tiempo).

Quinta etapa: Incorporación de las dimensiones al modelo de clima organizacional establecido en la primera etapa. En la quinta etapa también se siguió el método de Lawshe (1975) para la incorporación de las dimensiones al modelo de clima organizacional para las IES. Se incorporaron a cada factor, a través del consenso.

Las dimensiones reexpresadas para las IES, se integran al modelo de clima organizacional establecido en la primera etapa quedando con los factores personales con tres dimensiones (Motivación Intrínseca, Identidad y Autonomía) los factores grupales con cinco dimensiones (Trabajo en equipo, Apoyo, Administración del Conflicto, Respeto y Percepción de la organización) con cinco también lo factores organizacionales objetivos (Visión, Estructura, Comunicación, Condiciones de trabajo, Innovación). Es importante aclarar que las dimensiones utilizadas en el modelo de las IES coinciden en su mayor parte con las consideradas por Diana Guadalupe Garza Puente (2010) pero en el modelo utilizado se agregan además Estructura, Identidad, Respeto y Administración del conflicto.

Sexta etapa: revisión y adaptación de ítems e integración del instrumento. Una vez seleccionadas las dimensiones, se revisaron los ítems correspondientes a cada una de las dimensiones correspondientes en los instrumentos analizados, seleccionando los que al parecer de los investigadores deberían de incorporarse en la medición de cada una de las dimensiones del modelo de las IES y adaptando su redacción e incluyendo nuevos ítems. La elaboración de los ítems por expertos en la investigación sobre Clima Organizacional, está sustentada en cada uno de los estudios seleccionados y reseñados en la tercera etapa, lo que otorga al instrumento validez de contenido.

En total se redactaron 107 ítems, se integró el instrumento con 62 ítems con dirección positiva y 45 con dirección negativa. Correspondiendo a cada dimensión el número de ítem que se señalan en la tabla 5 , los ítems se ordenaron aleatoriamente en el instrumento. (Tabla 1)

La encuesta sobre Clima Organizacional de las IES, se elaboró en escala tipo Likert, ante cada ítem se presentaron cinco opciones: totalmente de acuerdo (TA), de acuerdo (A), neutral ( $N$ ), en desacuerdo (ED) y totalmente en desacuerdo (TD) 
Tabla 1. Número de ítems por dimensión y factor

\begin{tabular}{|c|c|c|c|}
\hline Factores & Dimensión & $\begin{array}{l}\text { Número de ítems } \\
\text { por dimensión }\end{array}$ & $\begin{array}{l}\text { Total de } \\
\text { ítems por } \\
\text { factor }\end{array}$ \\
\hline \multirow{3}{*}{ Factores personales } & Motivación intrínseca & 6 & \multirow{3}{*}{26} \\
\hline & Identidad & 9 & \\
\hline & Autonomía & 11 & \\
\hline \multirow{5}{*}{ Factores Grupales } & Trabajo en equipo & 6 & \multirow{5}{*}{22} \\
\hline & Ароуо & 5 & \\
\hline & Administración del conflicto & 3 & \\
\hline & Respeto & 6 & \\
\hline & Percepción de la organización & 2 & \\
\hline \multirow{5}{*}{$\begin{array}{c}\text { Factores } \\
\text { Organizacionales } \\
\text { objetivos }\end{array}$} & Visión & 3 & \multirow{5}{*}{27} \\
\hline & Estructura & 6 & \\
\hline & Comunicación & 7 & \\
\hline & Condiciones de trabajo & 5 & \\
\hline & Innovación & 6 & \\
\hline \multirow{6}{*}{$\begin{array}{c}\text { Factores } \\
\text { Organizacionales } \\
\text { Subjetivos }\end{array}$} & Reconocimiento & 6 & \multirow{6}{*}{32} \\
\hline & Salario & 4 & \\
\hline & Capacitación y desarrollo & 5 & \\
\hline & Promoción y carrera & 5 & \\
\hline & Equidad & 3 & \\
\hline & Presión & 9 & \\
\hline & Total & & 107 \\
\hline
\end{tabular}

\section{Aplicación y Registro}

Las siguientes etapas (séptima y octava) se relacionan con la aplicación de la prueba piloto, del instrumento a la población seleccionada y con la forma en que se registró la información.

Séptima etapa: prueba piloto. Una vez elaborado el instrumento, se tomó una pequeña muestra de maestros de una de las facultades, de la universidad pública donde se desarrolló la investigación, quienes respondieron libremente el cuestionario y realizaron observaciones al mismo, las que fueron incluidas para mejorar la versión final del instrumento.

Octava etapa: aplicación del instrumento en la IES, calificación de las respuestas e interpretación. Se logró que respondieran un total de 1289 académicos de la IES, que representan el 66\% de la población total. La información fue registrada en una base de datos previamente elaborada en excel, una vez recopilada la información se agrupó en forma general (los datos de toda la institución) y por unidad académica en SPSS 22.

Los ítems con dirección positiva se calificaron de la siguiente manera: $\mathrm{TA}=5$ puntos, $\mathrm{A}=4$ puntos, $\mathrm{N}=3$ puntos, $\mathrm{D}=2$ puntos y $\mathrm{TD}=1$ punto y los ítems con dirección negativa con valores contrarios. Se calcularon los valores promedio para cada dimensión en base a los ítems 
agrupados, y las puntuaciones del Clima Organizacional se interpretaron conforme una escala en donde: Muy malo de 1.0-1.8; Malo de 1.81-2.6; Regular de 2.61-3.4; Bueno de 3.41- 4.2 y Muy Bueno de 4.21- 5.0.

\section{Determinación de la consistencia Interna (Novena etapa)}

Con el apoyo del SPSS, se calculó el coeficiente de confiabilidad alfa de Cronbach para determinar la confiabilidad de la consistencia interna del instrumento y de cada uno de los factores considerados y se realizó prueba ANOVA para considerar si los factores podría ser considerados como grupos que miden variables diferentes obteniéndose los siguientes resultados.

Los valores obtenidos de alfa Cronbach tanto para el instrumento como para cada uno de los factores nos indica que la consistencia interna es alta ya que se tienen valores para los Factores Personales de 0.823; de 0.868 para los Factores Grupales; para los Factores organizacionales objetivos de 0.870; y de 0.876 para los Factores Organizacionales Subjetivos. y lo mismo se puede decir para el valor de alfa si el ítem es eliminado se encuentran todos por encima de 0.958 .

\section{Validez del Constructo (Décima Etapa)}

La evaluación del modelo elaborado, que incluye un factor más de los establecidos por Brunnet, se prueba por medio de ANOVA para conocer si existen diferencias significativas entre los factores encontrando los resultados que se reportan en la tabla 2.

Tabla 2. Prueba de ANOVA entre grupos

\begin{tabular}{cccccc}
\hline Evaluación & Suma de cuadrados & gl & $\begin{array}{c}\text { Media } \\
\text { cuadrática }\end{array}$ & F & Sig. \\
\hline Entre grupos & 188.780 & 3 & 62.927 & 218.790 & .000 \\
Dentro de grupos & 1481.771 & 5152 & .288 & & \\
Total & 1670.551 & 5155 & & & \\
\hline
\end{tabular}

Habiendo establecido la existencia de diferencia entre los grupos se realizó la evaluación de prueba t de Student para grupos aparejados, dando como resultado que todos los grupos son diferentes entre sí, esto es la división de los factores organizacionales en objetivos y subjetivos es válida. (Tabla 3)

Tabla 3. Prueba t para muestras apareadas

\begin{tabular}{cccc}
\hline Muestras apareadas & t & gl & $\begin{array}{c}\text { Sig. } \\
\text { (bilateral) }\end{array}$ \\
\hline Factores Personales - Factores Grupales & 24.634 & 1288 & 0.000 \\
Factores Personales - Factores Objetivos & 27.722 & 1288 & 0.000 \\
Factores Personales - Factores Subjetivos & 46.082 & 1288 & 0.000 \\
Factores Grupales - Factores Objetivos & -1.968 & 1288 & 0.049 \\
Factores Grupales - Factores Subjetivos & 28.281 & 1288 & 0.000 \\
Factores Objetivos - Factores Subjetivos & 30.388 & 1288 & 0.000 \\
\hline
\end{tabular}




\section{Conclusión}

El resultado es un instrumento para medir clima organizacional en IES, basado en un modelo de cuatro factores y 19 dimensiones, este modelo ha probado tener validez de contenido y consistencia interna.

El nivel de confiabilidad obtenido para la muestra, permite predecir un alto nivel de confiabilidad en la evaluación del clima en otras poblaciones, y aunque el número de ítems es alto permite un diagnóstico amplio en relación a cada dimensión y por factor que resulta especialmente útil cuando se trata de dar recomendaciones cuando el resultado no es bueno, y se pretenden implementar acciones específicas y efectivas en instituciones de educación superior. Como puntos pendientes queda someter los resultados el instrumento y sus constructos al análisis de pares y sus resultados a la comparación simultánea de otros instrumentos.

\section{References:}

1. Álvarez G.(1992). El clima en instituciones Educativas: conceptualización, investigaciones y resultados . Revista Interamericana de Psicología Ocupacional, (11), 1 y 2.

2. Acosta O. C., y López y Maldonado, J. (2010). Diagnóstico Clima Organizacional Colegio Gran Bretaña, Concepción. (Tesis de doctorado, inédita), Universidad de Playa Ancha de Ciencias de la Educación, Valparaíso, Chile.

3. Astudillo, C. (1985). Cuestionario para medir clima organizacional en instituciones financieras. (Tesis de Licenciatura). Escuela de Psicología, Pontificia Universidad Católica de Chile. Santiago, Chile.

4. Brunet, L. (1987). El clima de trabajo en las organizaciones. México: Trillas.

5. Cañellas Granda, J., Castellanos González, M., Piña Loyola, C. N., Yera Sánchez, A., Mir Ocampo, I. y Sánchez García, Z. (2007). Aspectos del clima organizacional en el Policlínico Universitario, Medisur, 5(3) 79-84.

6. Chiang Vega, M. (2004). Relación entre clima organizacional y satisfacción laboral en grupos de profesores y/o investigadores universitarios. (Tesis doctoral inédita), Universidad Pontificia Comillas de Madrid, Mdrid, España.

7. Chiang Vega M., Nuñez Partido A., y Huerta Rivera P. C. (septiembre-diciembre, 2007). Relación del clima organizacional y la satisfacción laboral con los resultados, en grupos de docentes de instituciones de educación superior ICADE. Revista cuatrimestral de las Facultades de Derecho y Ciencias Económicas y Empresariales, 72, 49-74. 
8. Chiang Vega M., Nuñez Partido A., y Huerta Rivera P. C. (2010). Efecto del clima organizacional en la autoeficacia de los docentes de instituciones de educación superior. Horizontes Empresariales, 19, 61-74.

9. Emig Klein L. M., Lazo Gumucio F. J. (2002). Clima organizacional: estamento académico de la Universidad Austral de Chile (Tesina de licenciatura inédita), Universidad Austral de Chile, Valdivia, Chile.

10. Dorman, J.P. (1998). The development and validation of an instrument to assess institutional-level environment in universities. Learning Environments Research, 1 (3), 333-352, Australia.

11. García Solarte M. (Julio-Diciembre, 2009). Clima Organizacional y su Diagnóstico: una aproximación conceptual. Cuadernos de Administración Universidad del Valle, 42, 43-61.

12. García Velázquez, M.R., Hernández Gracia, T. J., Navarrete Zorrilla, D. M., Corichi García, A. y Sánchez Monjaraz G. (October, 2014). Elementos de los modelos determinantes del clima organizacional. European Scientific Journal 10 (28), 1857- 7431.

13. Garza Puente, D. G. (2010). Análisis del clima organizacional. Caso de estudio de la Coordinación de Seguridad y Custodia del Centro de Ejecución de Sanciones de la Secretaría de Seguridad Pública en Tamaulipas (Tesis de maestría inédita), Universidad Autónoma de Tamaulipas, Tamaulipas, México.

14. Goncalvez A. (1997). Fundamento del Clima Organizacional. Sociedad Latinoamericana de Calidad (SLC), recuperado de: http://www.qualidade.org/articles/dec97/dec97.htm

15. Jones, A.P., \& James, L.R. (1979). Psychological climate: Dimensions and relationships of individual and aggregated work environment perceptions. Organizational Behavior and Human Performance, 23, 201-250

16. Lawshe, C.H. (December, 1975). A quantitative approach to content validity. Personnel Psychology, 28(4) 563-575. En Cohen, R. \& Swerdlik, M. (2001). Pruebas y evaluación psicológicas: Introducción a las pruebas y a la medición. México, D.F., México: Mc Graw Hill

17. Likert, R. (1961). New Patterns of Management. New York, USA: McGraw-Hill.

18. Likert, R. (1967). The Human Organization: Its Management and Value. New York, USA: McGraw-Hill.

19. Litwin, G.H. y Stringer, R.A. (1968). Motivation and organizational climate. Harvard University Graduate School of Business Administration, Boston: Press. 
20. Méndez, C. (2006). Clima Organizacional en Colombia. Un método de Análisis para su intervención. Facultad de Administración. Colección Lecciones. Universidad del Rosario. Bogotá

21. Mujica de González M., Pérez de Maldonado I. (Mayo-Agosto, 2007). Gestión del Clima Organizacional: una acción deseable en la Universidad. Laurus, 13 (24), 290-304.

22. Newman, J.E. (December 1977). Development of a Measure of Perceived Work Environment. The Academy of Management Journal, 20 (4), 520-534.

23. Payne, Dicker (1971). Organizational Structure and Climate, en M.D, Dunnette (ed), Hanbook of industrial and organizational psychology, Rand McNally, Chicago, Pág. 494.

24. Prisco Cortés X., Salaiza Lizárraga F. C. (Octubre de 2012). Relación entre el clima laboral y la motivación del personal en una secundaria pública. Memorias del XVII Congreso Internacional de Contaduría, Administración e Informática, Ciudad de México, México.

25. Redlich, J., y Trautmann, C. (1996). Un Estudio aplicado de clima organizacional en el estamento no académico de la Universidad Austral de Chile, sede Valdivia. (Tesis de licenciatura inédita). Universidad Austral de Chile, Valdivia, Chile.

26. Rodríguez, Darío (2005). Diagnóstico Organizacional. Bogotá, Colombia: Editorial Alfaomega

27. Schneider B. y Barttlet, C. J. (September, 1968) Individual differences and organizational climate: I. The research plan and questionnaire development. Personnel Psychology, (21)3, 323-333

28. Schneider, B. and Reichers, A. E. (March, 1983). On the etiology of climates. Personnel Psychology, 36, 19-39.

29. Silva, M. (Octubre - Diciembre, 1992). Hacia una definición comprehensiva del clima organizacional. Revista de Psicología General y Aplicada, 45(4), 443-451.

30. Salaiza Lizárraga F. C. (Mayo de 2008). Validez Factorial de la Encuesta de Clima Laboral de Ricardo Valenzuela. XII Congreso Anual de la Academia de Ciencias Administrativas, Tijuana, BC., México.

31. Sudarsky, J. (1977). Un Modelo de Diagnóstico e Intervención. Desarrollo Organizacional. Editorial Universitaria de América.

32. Toro, F. (2003). Incidencia del Clima Organizacional sobre el Apoyo organizacional Percibido. Revista Interamericana de Psicología Organizacional, 22 (2), 77-83.

33. Tunal Santiago G., Camarena Adame M. E., Pantón Romero I. (Agosto, 2007). Valoración desde la Teoría Social del Modelo de Clima Organizacional. Espacios Públicos, 10 (19), 45-61. 
34. Ucros Brito M. (Mayo-Agosto, 2011). Factores del clima organizacional en las universidades de la Costa Caribe Colombiana. Omnia, 17 (2), 91-102.

35. Valenzuela, J.R. (2004). Evaluación de Instituciones Educativas. México D.F., México: Trillas

36. Velázquez Ramírez D. (2012). Clima Organizacional en la Universidad Del Valle. (Documento de Trabajo Diagnóstico), Universidad del Valle, Cali, Colombia.

37. Vázquez Martínez R., y Guadarrama Granados J. J. (Enero-Julio, 2001). El clima organizacional en una organización tecnológica de educación superior. Tiempo de Educar, 3 (5), 105-131.

38. Vega D., Arévalo A., Sandoval J., Aguilar M. C., y Giraldo J. (JulioDiciembre, 2006). Panorama sobre los estudios de clima organizacional en Bogotá, Colombia (1994-2003). Diversitas: Perspectivas en Psicología, 2, 329-349.

39. Weick, K. E. (2009). Las organizaciones educativas como sistemas flojamente acoplados Gestión y Estrategia, 36(2). Caldera, D. y Ortega M. (Traducción libre) de Weick, Karl E. (Marzo, 1976). Educational Organizations as Loosely Coupled Systems, Administrative ScienceQuarterly, 21 (1), 1-19. 